MYU Tokyo

S \& M 1296

\title{
A Novel Method Based on a High-Dynamic Hybrid Forecasting Model for Fiber Optic Gyroscope Drift
}

\author{
Xiaowen Cai, Chunxi Zhang, Shuang Gao, ${ }^{*}$ Lu Wang, and Xianmu Li \\ Institute of Opto-Electronics Technology, Beihang University, \\ 37 Xue Yuan Road, Beijing 100191, China
}

(Received January 4, 2016; accepted June 3, 2016)

Keywords: fiber optic gyroscope drift, empirical mode decomposition model, adaptive residual grey model, improved autoregressive average, moving average

The drift of a fiber optic gyroscope (FOG) has a significant impact on the precision of an inertial navigation system (INS). In order to predict the FOG drift more efficiently, we have developed a method of reducing the drift using a hybrid-forecasting model. In the proposed model, the systematic and random parts of the FOG drift data are decomposed using the empirical mode decomposition (EMD) model. Then the systematic part is predicted by employing the adaptive residual grey model $[\operatorname{ARGM}(1,1)]$, and the random part is predicted by the improved autoregressive moving-average (IARMA) model. The final prediction results are the superimposition of the respective prediction using the EMD reconstruction model. The experimental results show that the gyroscope drift can be forecast precisely and can provide a basis for gyroscope performance analysis and fault prediction. At the same time, it can be concluded that the hybrid modeling has a higher forecasting precision than the single forecasting method.

\section{Introduction}

Inertial navigation systems (INSs) are widely used for navigation and positioning systems, and possess attractive features complementary to the global positioning system (GPS). Given inertial sensors, no external information other than initial pose estimation is required.(1) An INS can provide a good signal-to-noise ratio, especially in the case of rapid directional change and high rotational speed. ${ }^{(2,3)}$ As the gyroscope is the major component of an INS, the precision of an INS depends mainly on the gyroscope's precision. Therefore, the gyroscope's parameters are the main factors affecting the location and course angle accuracy in these navigation systems. ${ }^{(4)}$ The fiber optic gyroscope (FOG) is now at a very advanced stage of production and has significant advantages such as long life span, short warm-up time, high reliability, and wide dynamic range. ${ }^{(5)}$ In aviation, nautical, and terrestrial navigation applications, the precision of the application system is always restricted by the FOG drift.

To date, the application prospects for FOGs are very promising, and many methods have been developed to eliminate the drift.(6) The FOG drift can be classified into two types: systematic and random. ${ }^{(7)}$ The major systematic error sources include bias and scale factors. On the other hand, the random errors due to random drift primarily include sensor noise, which consists of two parts:

*Corresponding author: e-mail: shuanggao@126.com

http://dx.doi.org/10.18494/SAM.2017.1331

ISSN 0914-4935 C MYU K.K. 
a low-frequency component and a high-frequency component. ${ }^{(8)}$ A calibration test is generally used for the systematic part. For the random part, researchers have proposed many methods for filtering the high-frequency noise prior to processing, such as the use of a low-pass filter, a wavelet analysis, and a neural network denoising technique..$^{(9)}$ Wu et al. ${ }^{(10)}$ and Tian et al. ${ }^{(11)}$ used a Kalman filter (KF) to compensate FOG drift. Li et al. ${ }^{(12)}$ discussed the autoregressive moving-average (ARMA) model for reducing stochastic errors. Cui et al. ${ }^{(13)}$ presented a newly proposed hybrid filter called empirical mode decomposition-grey-forward linear prediction (EMD-G-FLP) to eliminate noise and extract the slowly varying drift.

In this study, we separated the systematic drift and random drift using the empirical mode decomposition (EMD) model, and proposed an adaptive residual grey model (ARGM) to decrease the systematic error. At the same time, we used an improved autoregressive moving-average (IARMA) model to reduce the random error. These algorithms shortened the compensation time and improved the FOG precision. The final predictive results were superimposed using the EMD reconstruction model. Experimental results show that the FOG drift was significantly reduced by the proposed method.

The remainder of this paper is organized as follows: Section 2 introduces the concept of the grey model [GM $(1,1)]$ and ARMA models. Section 3 describes the framework of the forecasting model and discusses the ARGM and IARMA model algorithms to compensate for the FOG drift. The simulation results are presented to confirm the effectiveness of the proposed method in Sect. 4, and our conclusions are stated in Sect. 5.

\section{Theoretical Background}

\subsection{GM $(1,1)$ overview}

Grey system theory, as proposed by Deng, is the simplest and most common unknown data forecasting model. ${ }^{(10,14)}$ In recent decades, it has been widely applied in various research fields.

For the original series of FOG output $x^{(0)}(i), i=1,2, \ldots, n$, a new series can be generated by the first-order accumulated generating operation (1-AGO) as

$$
x^{(1)}(k)=A G O\left(x^{(0)}(k)\right)=\sum_{i=0}^{k} x^{(0)}(i),
$$

where $x^{(1)}(1)=x^{(0)}(1), k=2,3, \ldots, n$.

From $x^{(1)}(k)$, we can form the grey prediction model GM $(1,1)$ from which the modeling value is obtained as

$$
\hat{x}^{(1)}(k)=\left(x^{(0)}(1)-\frac{b}{a}\right) e^{-a(k-1)}+\frac{b}{a},
$$

where $a$ denotes the grey developmental coefficient and $b$ represents the grey control parameter, $k=2$, $3, \ldots, n$. 
The values of parameters $a$ and $b$ can be estimated by the least-squares method (LSD), that is,

$$
\left[\begin{array}{ll}
a & b
\end{array}\right]^{\mathrm{T}}=\left(B^{\mathrm{T}} B\right)^{-1} B^{\mathrm{T}} y_{n}
$$

where

$$
B=\left[\begin{array}{cc}
-0.5\left(x^{(1)}(1)+x^{(1)}(2)\right) & 1 \\
-0.5\left(x^{(1)}(2)+x^{(1)}(3)\right) & 1 \\
\vdots & \vdots \\
-0.5\left(x^{(1)}(n-1)+x^{(1)}(n)\right) & 1
\end{array}\right]
$$

and

$$
y_{n}=\left[\begin{array}{llll}
x^{(0)}(2) & x^{(0)}(3) & \cdots & x^{(0)}(n)
\end{array}\right]^{\mathrm{T}} .
$$

Applying the first-level inversed AGO (1-IAGO) to $\hat{x}^{(0)}(k+1)$, the forecast fitting value of the raw displacement sequence of the FOG can be expressed as

$$
\left\{\begin{array}{l}
\hat{x}^{(0)}(k+1)=\hat{x}^{(1)}(k+1)-\hat{x}^{(1)}(k) \\
\hat{x}^{(0)}(1)=\hat{x}^{(1)}(1)
\end{array}\right.
$$

Usually, GM $(1,1)$ is used as a short-term prediction scheme because Eq. (3) only requires two coefficients to be identified.

\subsection{ARMA model}

The ARMA model is the most basic sequential method and a practical application of the most comprehensive time-series model.(15) It expands and develops the linear regression model foundation. The ARMA model can promulgate the structure of dynamic data and the rule that will forecast its future value.

Assume that $\left\{x_{t}, t=0, \pm 1, \pm 2, \ldots\right\}$ is a stationary time series with a zero mean that forms the random part of the FOG drift. The generalized form of the ARMA model can be described as

$$
\varphi(B) x_{t}=\theta(B) \varepsilon_{t},
$$

where $\varphi(B)=1-\varphi_{1} B-\varphi_{2} B^{2}-\cdots-\varphi_{p} B^{p}$ and $\theta(B)=1-\theta_{1} B-\theta_{2} B^{2}-\cdots-\theta_{q} B^{q}$, and $B$ denotes the backward shift operator, $\varepsilon_{t}$ is stationary white noise with a zero mean, $p$ and $q$ are the orders of the ARMA model, and $\varphi_{i}(i=1, \ldots, p)$ and $\theta_{i}(i=1, \ldots, q)$ are the parameters of the ARMA model. When $p=0$, the $\operatorname{ARMA}(p, q)$ model is the autoregressive $[\operatorname{AR}(p)]$ model. When $q=0$, the $\operatorname{ARMA}(p, q)$ model is the moving-average [MA $(q)]$ model. The ARMA model can also be described as

$$
x(k)=-\sum_{i=1}^{p} \varphi_{i} x(k-i)+\sum_{j=1}^{q} \theta_{j} x(k-j) .
$$




\section{Improving the Error Model}

Although GM $(1,1)$ is useful for predictions with less data and easy calculations, it is not suitable for more complicated undulant data. Furthermore, if the data has a small variation regularity then serious errors may occur. Thus, GM $(1,1)$ must be improved to increase the prediction accuracy. On the other hand, the establishment of the ARMA model requires accurate model identification, because false model identification will cause the wrong stage of model estimation and increase the cost of re-identification. ${ }^{(16)}$

In order to increase the precision of the FOG, an improved method is proposed, as per the algorithm shown in Fig. 1. The method has the following several steps.

Step 1: Data preprocessing: In view of the highly dynamic FOG drift phenomenon, EMD is used to decompose the drift data of the FOG, and separate the systematic drift and the random drift from the original data. The decomposition criterion is that the low-frequency signal and the original signal trends are similar, and the high-frequency signal is similar to a stationary signal.

Step 2: Model building: The ARGM $(1,1)$ and IARMA models are used to predict the systematic and random parts of the FOG drift, respectively, and the prediction results are obtained.

Step 3: Prediction evaluation. The final prediction result is the superimposition of the respective prediction made using the EMD reconstruction model.

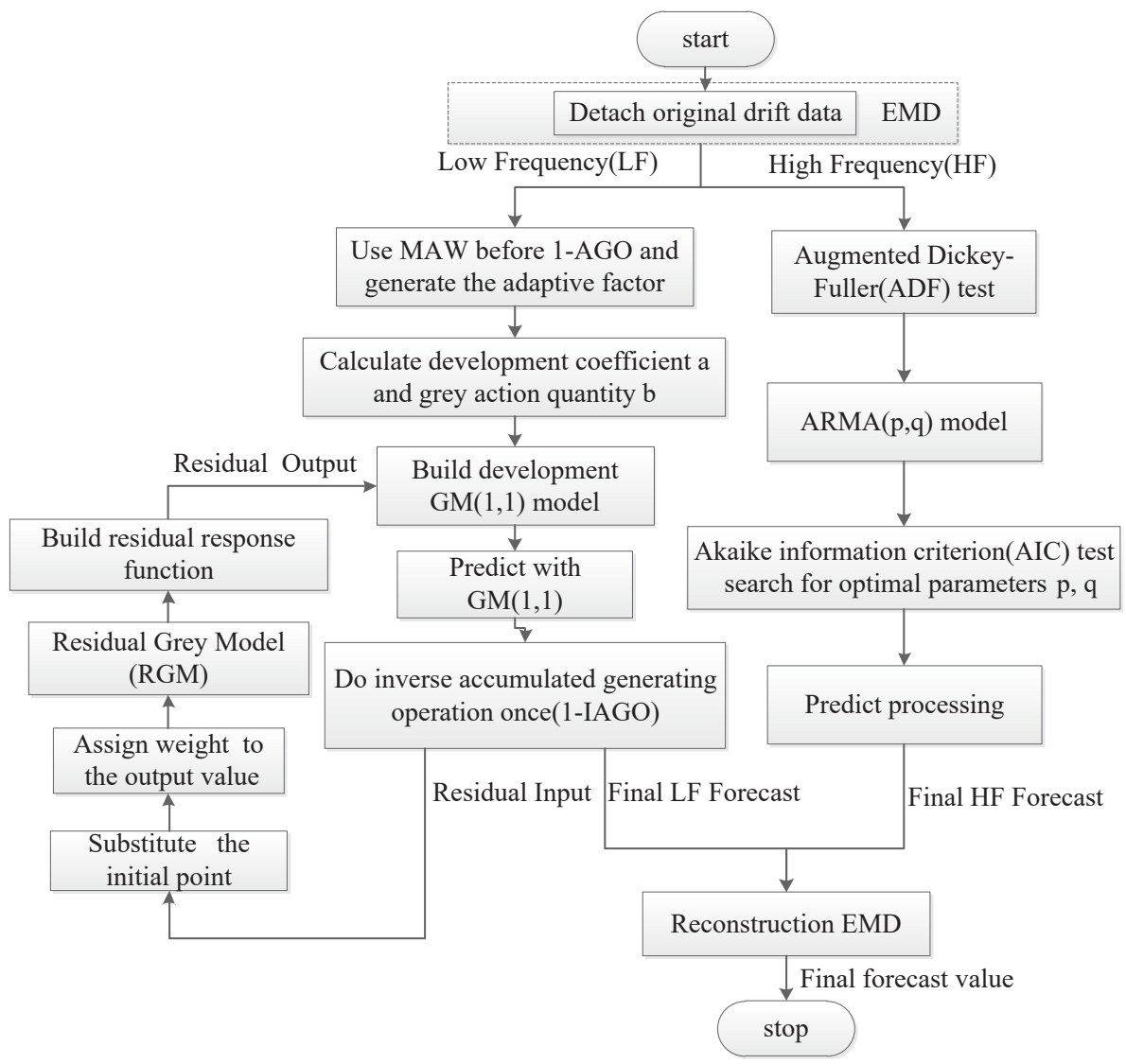

Fig. 1. Schematic of the model algorithm. 
The ARGM $(1,1)$ and IARMA models are the most important steps of the algorithm, and will be introduced in the next two sections.

\subsection{Design of the ARGM $(1,1)$ model}

Most researchers have improved the model so that the background noise values are improved.(17) A few studies have enhanced the predictive accuracy of multiple aspects, such as the background and original values. $^{(18)}$ In this study, we use $\operatorname{ARGM}(1,1)$ based on the RGM, ${ }^{(19)}$ which uses the moving-average windows (MAW) method to deal with the raw data points of the FOG $\hat{x}^{(0)}(i),(i=1,2$, $\ldots, n)$. First, it uses an accumulation process to deal with the original data, and then uses the MAW method to deal with the entire number sequence. The equation to deal with the sequence is

$$
\hat{x}^{(0)}(m)=\frac{\hat{x}^{(0)}(m-1)+2 \hat{x}^{(0)}(m)+2 \hat{x}^{(0)}(m+1)+\hat{x}^{(0)}(m+2)}{6},
$$

where $m=2, \ldots, n-2$, and

$$
\begin{gathered}
\hat{x}^{(0)}(1)=\frac{3 \hat{x}^{(0)}(1)+2 \hat{x}^{(0)}(2)+\hat{x}^{(0)}(3)}{6}, \\
\hat{x}^{(0)}(n-1)=\frac{3 \hat{x}^{(0)}(n-2)+2 \hat{x}^{(0)}(n-1)+\hat{x}^{(0)}(n)}{6},
\end{gathered}
$$

and

$$
\hat{x}^{(0)}(n)=\frac{5 \hat{x}^{(0)}(n-1)+\hat{x}^{(0)}(n)}{6} .
$$

The use of the MAW method weakens the effect of the abnormal data points and improves the accuracy of the results.

We then substitute $\left\{\left(m, x^{(1)}(m)\right), m=1,2, \ldots, n\right\}$ for the initial point $\left(1, x^{(1)}(1)\right)$ and the modified $\operatorname{RGM}(1,1)$ formula becomes

$$
\hat{x}_{m}^{(1)}(k+1)=\left(x^{(1)}(m)-\frac{b}{a}\right) e^{-a k}+\frac{b}{a} .
$$

The ARGM $(1,1)$ output value is then

$$
\hat{x}_{M}^{(1)}(k+1)=c+\sum_{m=1}^{n} \lambda_{m} \hat{x}_{m}^{(1)}(k+1)
$$

where $\hat{x}_{M}^{(1)}(k+1)$ represents the predicted output value from $\operatorname{ARGM}(1,1), \lambda_{m}$ is the weighting factor, and $c$ denotes the adaptive factor to the output value, $m=1,2, \ldots, n$.

If quasi-smooth checking $0 \leq x_{M}^{(0)}(k+1) / x_{M}^{(1)}(k) \leq 0.5$ is satisfied, then $c=0$. 
If $0 \leq x_{M}^{(0)}(k+1) / x_{M}^{(1)}(k) \leq 0.5$ is unsatisfied, then $c \geq \max \left\{\frac{2 x_{M}^{(1)}(k+1)-\sum_{i=1}^{k} x_{M}^{(1)}(i)}{k-2}\right\}$.

$\varepsilon^{(0)}(k)$ is then proposed as a residual sequence:

$$
\varepsilon^{(0)}(k)=\hat{x}^{(0)}(k)-x^{(0)}(k),
$$

where $k=1,2, \ldots, n$.

According to Eq. (15), if we substitute the subsequence numbers $\varepsilon^{(0)}\left(k^{\prime}\right)$, where $k^{\prime} \leq n$, for $\varepsilon^{(0)}(k)$ and establish GM $(1,1)$ on $\varepsilon^{(0)}\left(k^{\prime}\right)$, then $\hat{\varepsilon}^{(0)}\left(k^{\prime}+1\right)$ can be obtained as

$$
\hat{\varepsilon}^{(0)}\left(k^{\prime}+1\right)=\left(1-e^{a}\right)\left[\varepsilon^{(0)}\left(1^{\prime}\right)-\frac{b}{a}\right] e^{-a k^{\prime}} .
$$

The residual correction GM $(1,1)$ model for $\hat{\varepsilon}^{(0)}\left(k^{\prime}+1\right)$ is then expressed by

$$
\hat{x}^{(0)}\left(k^{\prime}+1\right)=\left(1-e^{a}\right)\left[x^{(0)}(1)-\frac{b}{a}\right] e^{-a k}+\delta(k-i)\left(1-e^{a}\right)\left[\varepsilon^{(0)}\left(1^{\prime}\right)-\frac{b}{a}\right] e^{-a k^{\prime}},
$$

where $\delta(k-i)= \begin{cases}1, & k \geq i, \\ 0, & k<i .\end{cases}$

Finally, the residual corrected value $\hat{x}^{(0)}(k+1)$ of the original sequence becomes

$$
\hat{\hat{x}}^{(0)}(k+1)=\hat{x}^{(0)}(k+1)+\hat{\varepsilon}^{(0)}\left(k^{\prime}+1\right) .
$$

Data were collected from the FOG for $13 \mathrm{~h}$ in accordance with the $\operatorname{RGM}(1,1)$ and $\operatorname{ARGM}(1,1)$ models. In order to simplify the figure, we take an average of the data over one hour for each of 13 sample points. The results are shown in Fig. 2.

As shown in Fig. 2, using ordinary $\operatorname{RGM}(1,1)$ and $\operatorname{ARGM}(1,1)$ for long-term forecasting, the results can be calculated, as shown in Table 1 .

From Table 1, we can see that the average of the relative error of the forecasting result from RGM (1, 1) is $3.77 \%$. However, the average of the relative error of the forecasting result from $\operatorname{ARGM}(1,1)$ is only $2.22 \%$, which is lower than the result of $\operatorname{RGM}(1,1)$. Thus, $\operatorname{ARGM}(1,1)$ obtains a higher accuracy of prediction.

\subsection{Modeling of IARMA}

We propose an improved model-building method based on the Augmented Dickey-Fuller (ADF) test and the Akaike Information Criterion (AIC) test.

The $\mathrm{ADF}$ test ${ }^{(20)}$ is a popular technique in engineering and was used to test the stationary element in the time-series data sets. In order to illustrate how the ADF test functions, we consider the simple $\operatorname{ARMA}(2,1)$ process below referring to Eq. (8).

$$
x(k)=-\varphi_{1} x(k-1)+\theta_{1} x(k-1)+\theta_{2} x(k-2)
$$




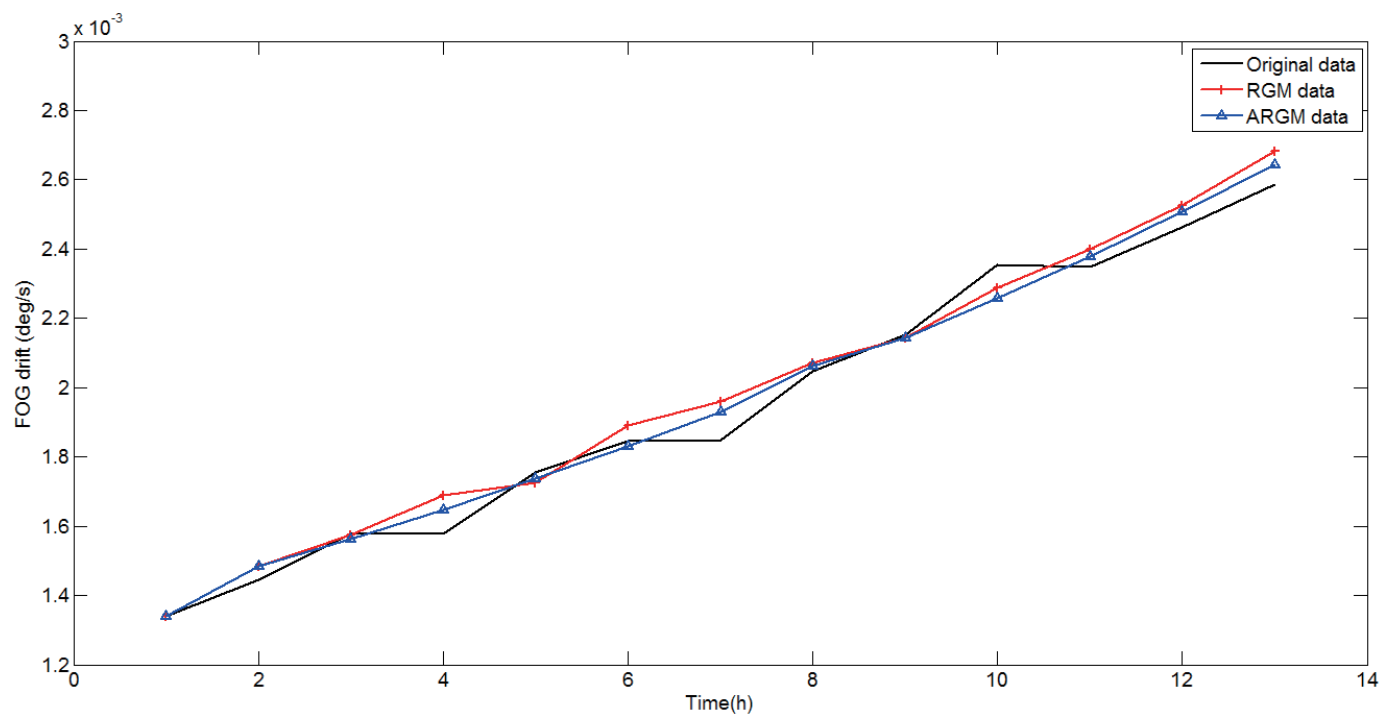

Fig. 2. (Color online) The curves of $\operatorname{RGM}(1,1)$ and $\operatorname{ARGM}(1,1)$.

Table 1

RMSE results for different data types.

\begin{tabular}{|c|c|c|c|c|c|}
\hline \multirow[b]{2}{*}{ Times } & \multirow[b]{2}{*}{$\begin{array}{l}\text { Actual data } \\
(\text { deg/h) }\end{array}$} & \multicolumn{2}{|c|}{$\operatorname{RGM}(1,1)$} & \multicolumn{2}{|c|}{$\operatorname{ARGM}(1,1)$} \\
\hline & & $\begin{array}{l}\text { Forecast data } \\
\quad(\mathrm{deg} / \mathrm{h})\end{array}$ & $\begin{array}{c}\text { Relative error } \\
(\%)\end{array}$ & $\begin{array}{l}\text { Forecast data } \\
(\mathrm{deg} / \mathrm{h})\end{array}$ & $\begin{array}{c}\text { Relative error } \\
(\%)\end{array}$ \\
\hline 1 & $1.3389 \times 10^{-3}$ & $1.34854 \times 10^{-3}$ & 0.723755 & $1.33975 \times 10^{-3}$ & 0.067222 \\
\hline 2 & $1.4445 \times 10^{-3}$ & $1.48360 \times 10^{-3}$ & 2.709663 & $1.48360 \times 10^{-3}$ & 2.709663 \\
\hline 3 & $1.5779 \times 10^{-3}$ & $1.57354 \times 10^{-3}$ & -0.27379 & $1.56354 \times 10^{-3}$ & -0.907559 \\
\hline 4 & $1.5779 \times 10^{-3}$ & $1.68778 \times 10^{-3}$ & 6.966398 & $1.64778 \times 10^{-3}$ & 4.431318 \\
\hline 5 & $1.7567 \times 10^{-3}$ & $1.72656 \times 10^{-3}$ & -1.70676 & $1.73656 \times 10^{-3}$ & -1.137463 \\
\hline 6 & $1.8471 \times 10^{-3}$ & $1.89013 \times 10^{-3}$ & 2.332368 & $1.83013 \times 10^{-3}$ & -0.916055 \\
\hline 7 & $1.8474 \times 10^{-3}$ & $1.95873 \times 10^{-3}$ & 6.028029 & $1.92873 \times 10^{-3}$ & 4.404099 \\
\hline 8 & $2.0473 \times 10^{-3}$ & $2.07265 \times 10^{-3}$ & 1.2401942 & $2.06265 \times 10^{-3}$ & 0.751737 \\
\hline 9 & $2.1536 \times 10^{-3}$ & $2.14217 \times 10^{-3}$ & -0.529816 & $2.14217 \times 10^{-3}$ & -0.529815 \\
\hline 10 & $2.3548 \times 10^{-3}$ & $2.28759 \times 10^{-3}$ & -2.85582 & $2.25759 \times 10^{-3}$ & -4.129792 \\
\hline 11 & $2.3474 \times 10^{-3}$ & $2.39923 \times 10^{-3}$ & 2.2101519 & $2.37923 \times 10^{-3}$ & 1.358127 \\
\hline 12 & $2.4619 \times 10^{-3}$ & $2.52742 \times 10^{-3}$ & 2.6630271 & $2.50742 \times 10^{-3}$ & 1.850633 \\
\hline 13 & $2.5851 \times 10^{-3}$ & $2.68252 \times 10^{-3}$ & 3.7705267 & $2.64252 \times 10^{-3}$ & 2.223168 \\
\hline
\end{tabular}

Note that this is the same as

$$
x(k)=\left(\theta_{1}-\varphi_{1}+\theta_{2}\right) x(k-1)-\theta_{2}(x(k-1)-x(k-2)),
$$

and subtracting $x(k-1)$ from both sides gives

$$
\Delta x(k)=\vartheta x(k-1)+\zeta_{1} \Delta x(k-1),
$$

where the following have been defined: 


$$
\left\{\begin{array}{l}
\vartheta=\theta_{1}-\varphi_{1}+\theta_{2} \\
\zeta_{1}=-\theta_{2}
\end{array}\right.
$$

After performing the ADF test on the IAMAR model prediction, we used $\Delta x(k)$ to compensate the ARMA model prediction. Then we used an automatic forecasting subprogram that executes data after differentiation to find the most accurate time-series model by finding the minimum AIC value. (21) The AIC test is one of the best techniques of fitting an estimated statistical model ${ }^{(22)}$ and of attempting to find a model to best explain the data with minimum free parameters.

The proposed AIC test is then used to obtain an estimate of the model order. The AIC test ${ }^{(23)}$ is given by

$$
A I C_{p, q}=-2(\log L)+2(p+q) .
$$

To determine the log-likelihood, the ARMA $(p, q)$ model parameters must be estimated for each model-order pair $(p, q)$. The EM algorithm ${ }^{(24)}$ is used to obtain an estimate of $(p, q)$ and ensure that the ARMA parameters are optimally estimated to prevent bias of the results of the AIC test. However, the model-order estimation through AIC is consequently much more computationally intensive than it was previously.

In short, we can achieve a combination forecasting model, which is composed of the ARGM $(1,1)$ model and the IARMA $(n, m)$ model:

$$
\hat{x}_{\text {total }}^{(0)}(k+1)=\hat{x}_{G}^{(0)}(k+1)+\hat{x}_{A}^{(0)}(k+1),
$$

where $\hat{x}_{G}^{(0)}(k+1)$ is the predictive output value calculated from $\operatorname{ARGM}(1,1)$, and $\hat{x}_{A}^{(0)}(k+1)$ is the value calculated from the IARMA model.

\section{Simulation and Results}

The drift data used in this study was provided by the Institute of Opto-electronics, Beihang University in Beijing, China. The FOG was tested repeatedly for $10 \mathrm{~h}$ and 360000 data points were recorded.

\subsection{Data preprocessing by EMD}

EMD was applied to decompose the original drift data of the FOG. The results from the original data, the high-frequency data from the random drift, the low-frequency data from the constant drift, and the residual data are shown in Fig. 3.

In Fig. 3, the residual error is in the sine form because EMD suffers from the end effect and a mode mixing problem. The end effect is a phenomenon that occurs at both ends of the data. We have not discussed these two problems in this paper. The root mean square error (RMSE) for each approximated component was calculated, and the results are presented in Table 2.

\subsection{Reducing drift in the FOG by ARGM $(1,1)$ and IARMA}

After decomposing the original drift, we used ARGM $(1,1)$ and IARMA to process the systematic and random drifts, respectively. 


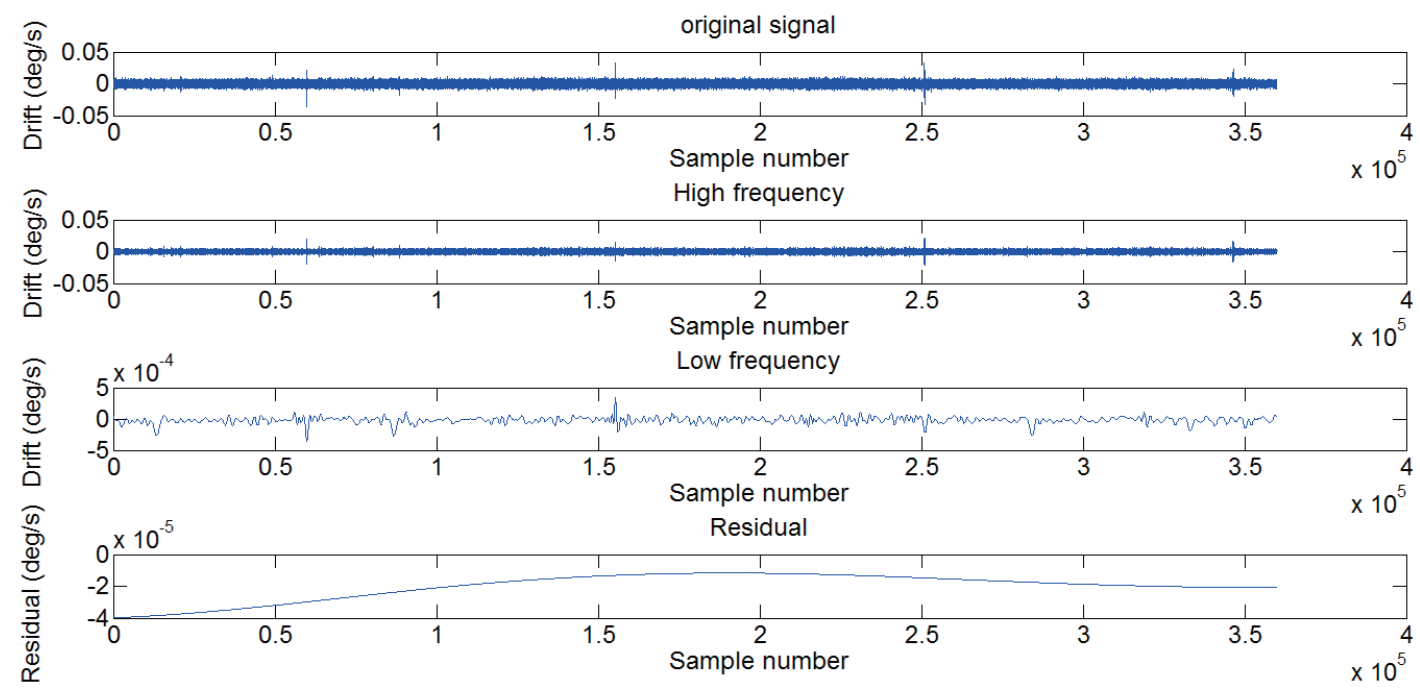

Fig. 3. (Color online) Drift data from the FOG.

Table 2

RMSE results for different data types.

\begin{tabular}{lccc}
\hline Data type & Original data & HF data & LP data \\
\hline RMSE $(\mathrm{deg} / \mathrm{s})$ & $2.77 \times 10^{-3}$ & $2.318 \times 10^{-3}$ & $6.126 \times 10^{-6}$ \\
\hline
\end{tabular}
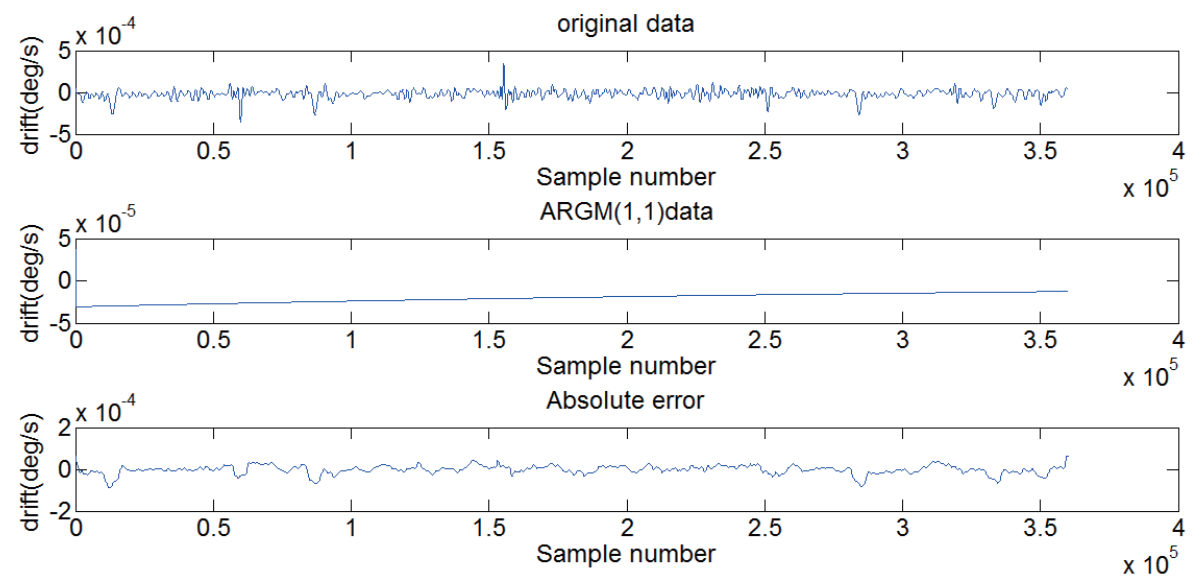

Fig. 4. (Color online) Original data, the $\operatorname{ARGM}(1,1)$ prediction, and the absolute error.

The results of the analysis using ARGM $(1,1)$ are shown in Fig. 4. One can see that absolute error refers to the difference between the original data and the predicted data from ARGM $(1,1)$. After ARGM $(1,1)$ processing, the low-frequency part of the original data is significantly improved.

Preprocessing of the random drift was performed simultaneously with the calculation of the autocorrelation function of the sample sequence, and a partial autocorrelation function was drawn. The results of the autocorrelation function of the sample sequences and the partial autocorrelation functions are shown in Fig. 5. Both can be seen to tail-off at low values of drift. 

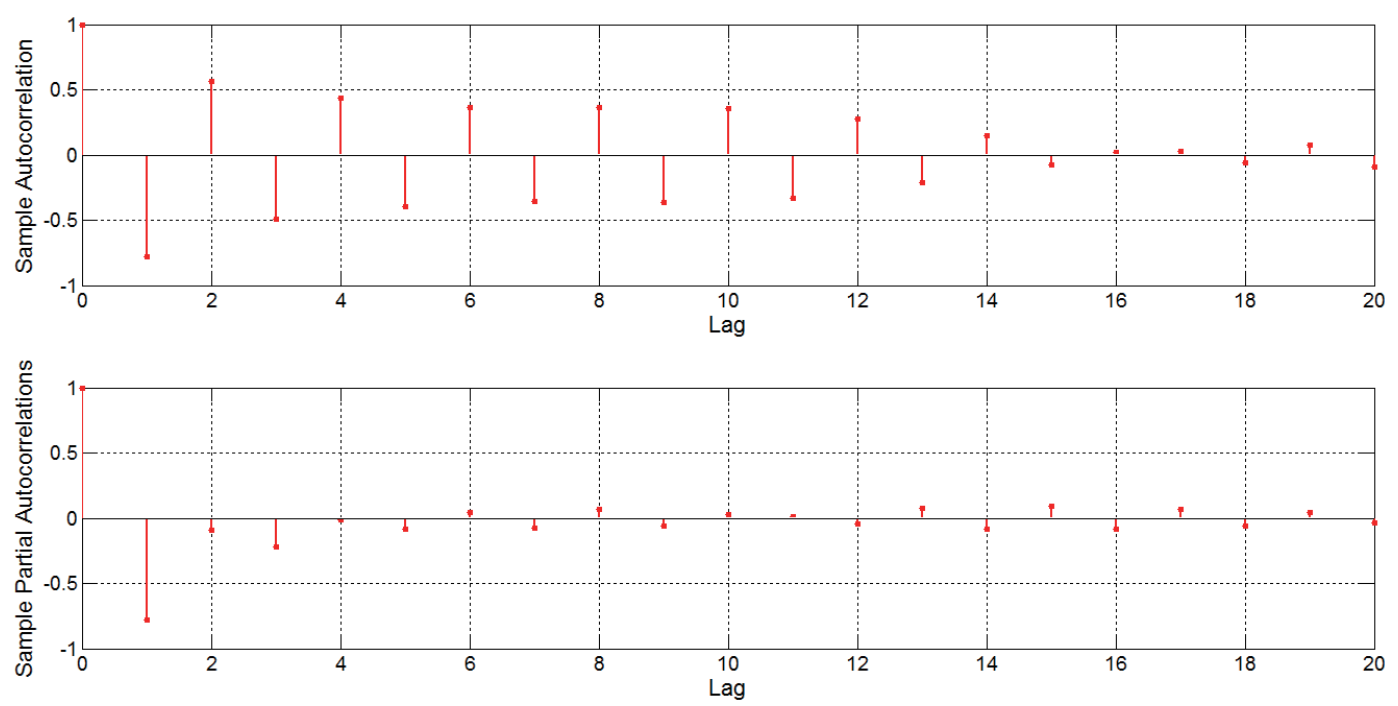

Fig. 5. (Color online) Results of the autocorrelation function and the partial autocorrelation function.

The calculation produces the values $p=2$ and $q=1$ by measuring the equivalent IARMA with the AIC test. The final equation for the FOG random drift is thus

$$
\hat{x}_{A}^{(0)}(k+1)=-0.2831 x_{k}-0.0912 x_{k-1}+\alpha_{k+1}-0.8769 \alpha_{k},
$$

where $\alpha_{k} \sim N\left(0,1.26 \times 10^{-5}\right)$.

The original data and predicted data are shown together in Fig. 6 for comparison. The peak position and the average amplitude are all decreased after IARMA processing.

Using the root mean square error (RMSE) for the IARMA $(2,1)$ model and the original data yielded the results shown in Table 3.

The similarity of the data shown in Fig. 6 demonstrates that this algorithm is suitable and has an accurate forecasting effect. The RMSE results in Table 3 also indicate that the IARMA model has high accuracy.

\subsection{Reconstruction of data}

The results of applying the new algorithm for data reconstruction using EMD are shown in Fig. 7. The RMSEs of the predicted data and the original data are shown in Table 4.

\subsection{Analysis and results}

The ARGM $(1,1)$ and IARMA models were applied to processing the drift data of FOGs in a similar manner to the procedure shown in Figs. 3-7. The resultant predicted curve of the FOG drift output using reconstruction by EMD is shown in Fig. 7. Clearly, the reconstruction using EMD yielded a better performance than the original one. 

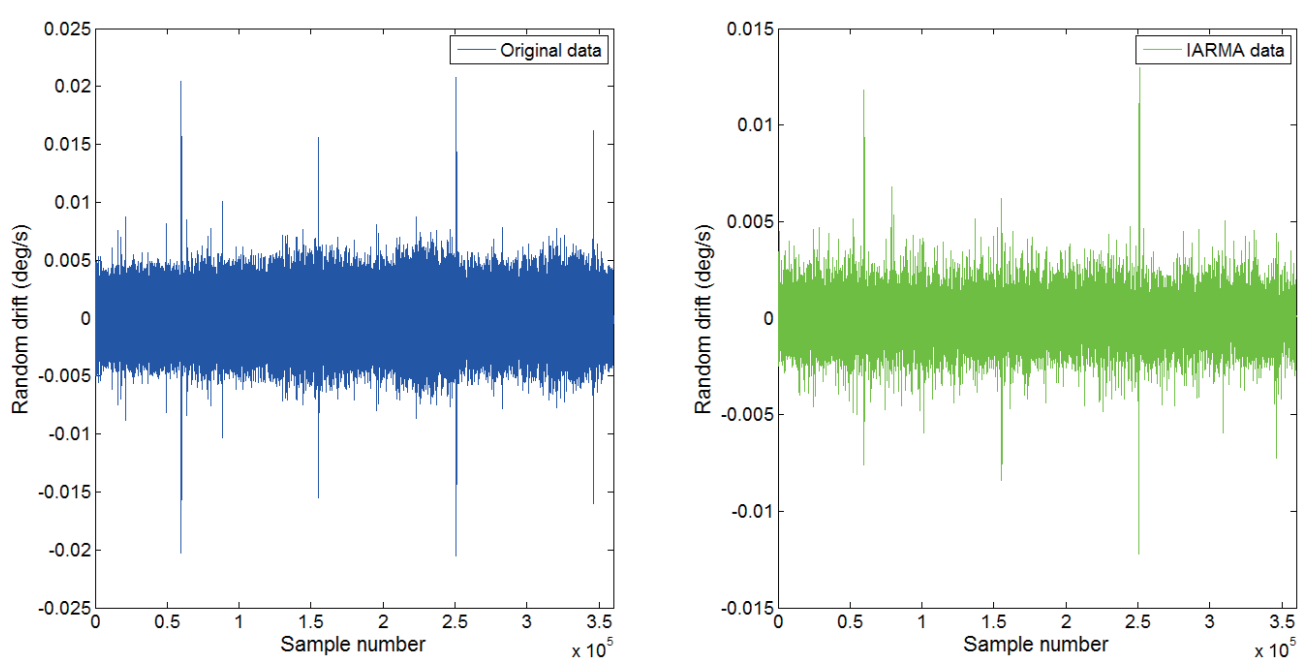

Fig. 6. (Color online) Predicted data and original data.

Table 3

RMSE results for the IARMA $(2,1)$ data and the original data.

\begin{tabular}{lcc}
\hline Data type & Original data & IARMA $(2,1)$ data \\
\hline RMSE $(\mathrm{deg} / \mathrm{s})$ & $2.77 \times 10^{-3}$ & $6.006 \times 10^{-4}$ \\
\hline
\end{tabular}
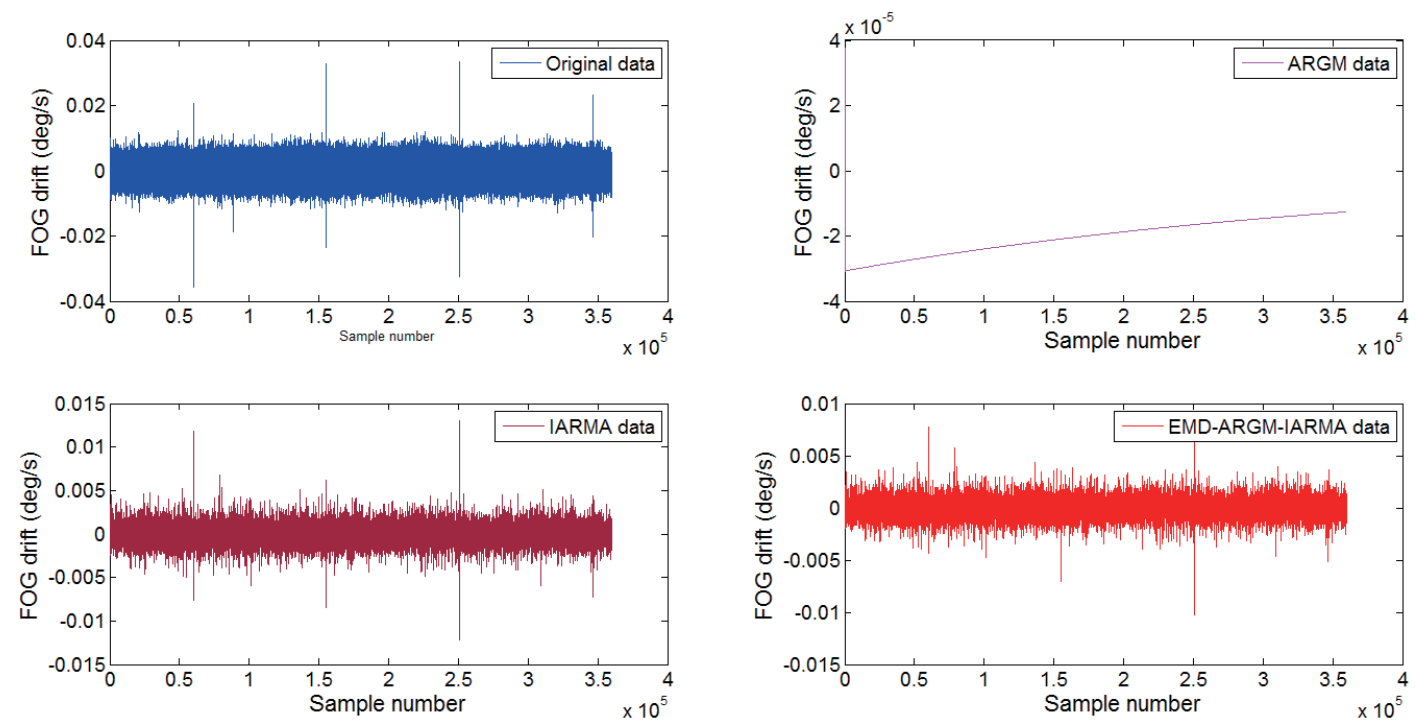

Fig. 7. (Color online) The final data predictions.

Table 4

RMSE results for the EMD-ARGM-IARMA data and the original data.

Data type Original data IARMA data EMD-ARGM-IARMA data

\begin{tabular}{llll}
\hline RMSE $(\mathrm{deg} / \mathrm{s})$ & $2.77 \times 10^{-3}$ & $6.006 \times 10^{-4}$ & $4.969 \times 10^{-4}$
\end{tabular}


Table 5

RMSEs of three methods.

\begin{tabular}{lccc}
\hline Data type & Original data & Proposed method data & Prediction precision (\%) \\
\hline Ref. 25 & -0.0271 & 0.0169 & 1.6 \\
& $(1$ step $)$ & $(4$ steps $)$ & \\
Ref. 26 & 0.9437 & 0.9416 & 0.82 \\
Our method (deg/s) & $2.77 \times 10^{-3}$ & $4.969 \times 10^{-4}$ & 0.05 \\
\hline
\end{tabular}

Table 6

Error coefficients of different drift noise factors.

\begin{tabular}{lccccc}
\hline Error coefficient & $\mathrm{QN}(\mu \mathrm{rad})$ & $\mathrm{BI}\left({ }^{\circ} / \mathrm{h}\right)$ & $\mathrm{ARW}\left({ }^{\circ} / \mathrm{h}^{1 / 2}\right)$ & $\mathrm{RRW}\left({ }^{\circ} / \mathrm{h}^{2}\right)$ & $\mathrm{ARR}\left({ }^{\circ} / \mathrm{h}^{3 / 2}\right)$ \\
\hline Original data & 0.5511 & 0.9356 & $3.655 \times 10^{-4}$ & $4.283 \times 10^{-4}$ & $9.947 \times 10^{-4}$ \\
Processed data & 0.0135 & 0.2626 & $9.348 \times 10^{-5}$ & $5.243 \times 10^{-5}$ & $9.047 \times 10^{-5}$ \\
\hline
\end{tabular}

The results obtained by Lu et al. ${ }^{(25)}$ and Zhou et al. ${ }^{(26)}$ are listed in Table 5 for comparison. The data in Table 5 show that the method discussed in this paper has a higher precision than the other two methods.

Furthermore, the Allan variance estimation method ${ }^{(27)}$ was adopted here to analyze the FOG drift data. The five basic noise terms in an FOG are quantization noise, bias instability, angle random walk, rate random walk, and rate ramp, with error coefficients represented by QN, BI, ARW, RRW, and ARR, respectively. The error coefficients of the remaining noise in FOG drift data are listed in Table 6.

The results in Table 6 clearly show that the level of noise in the FOG static output data after reconstruction using EMD was lower than that in the case of the original model.

\section{Conclusions}

The FOG is gaining increasing popularity because of its excellent performance. Reducing the drift of a FOG is the key to the performance of the entire INS system. To reduce FOG drift, we studied the use of the ARGM $(1,1)$ and IARMA models to process the systematic and random drifts, respectively. We eventually achieved reconstruction using EMD by combining the strength of the two models. In addition, we used the Allan variance to estimate the drift data for a FOG. Although processing the drift of FOGs in this way does not use the latest techniques, we have improved the accuracy of the algorithm significantly. Numerical results demonstrate that ARGM $(1,1)$ could overcome the drawbacks of using $\operatorname{RGM}(1,1)$ and also markedly increase the FOG accuracy and adaptability. Future work will focus on the effect of the proposed model when applied in different environments.

\section{Acknowledgement}

This work was supported by the National Key Scientific Instrument and Equipment Development Project (2013YQ50791). 


\section{References}

1 Y. Ren and X. Ke: Int. Inf. Manage. 2 (2010) 417.

2 P. Lang, A. Kusej, A. Pinz, and G. Brasseur: Proc. 2002 IEEE Instrumentation and Measurement Technology Conference (IEEE, 2002) p. 1583.

3 E. Marchand, P. Bouthemy, and F. Chaumette: Image Vision Comput. 19 (2001) 941.

4 F. Sun: Syst. Eng. Electron. 29 (2007) 1532.

5 K. T. V. Grattan and T. Sun: Sens. Actuators, A 82 (2000) 40.

6 K. Bohnert, S. Wildermuth, and H. Brandle: OSA/CLEO/IQEC 1 (2009) 32.

7 S. Nassar, K. Schwarz, N. El-Sheimy, and A. Noureldin: Navigation 51 (2004) 259.

8 J. Skaloud, A. Bruton, and K. Schwarz: Navigation 46 (1999) 97.

9 M. El Diasty and S. Pagiatakis: J. Global Pos. Syst. 7 (2008) 170.

10 X. Wu, L. Duan, and W. Chen: Proc. 2011 6th IEEE Industrial Electronics and Applications Conference (IEEE, 2011) p. 1933.

11 Y. Tian, X. Yang, Y. Guo, and F. Liu: Opt. Fiber Sens. Appl. 5 (2015) 223.

12 Y. Li, X. Xu, and C. Chen: Control Decis. 30 (2015) 934.

13 B. Cui, and X. Chen: Sens. Actuators, A 230 (2015) 150.

14 J. Deng: J. Grey Syst. 1 (1989) 24.

15 Y. Huang, Q. Wang, and Y. Li: Stat. Decis. 16 (2009) 210.

16 C. S. Ong, J. J. Huang, and G. H. Tzeng: Appl. Math. Comput. 164 (2005) 885.

17 Y. Zhang, Y. Wei, and C. Xiong: Syst. Eng. Theory Pract. 27 (2007) 141.

18 X. Liu, H. Peng, Y. Bai, Y. Zhu, and L. Liao: Procedia Behav. Sci. 138 (2014) 767.

19 C. Dai, D. Pi, Z. Fang, and H. Peng: IEEE Sens. J. 14 (2014) 1886.

20 D. A. Dickey and W. A. Fuller: J. Am. Stat. Assoc. 74 (1979) 427.

21 H. Akaike: IEEE Trans. Autom. Control 19 (1974) 716.

22 S. Halim, I. N. Bisono, Melissa, and C. Thia: Manage. Sci. Eng. Manage. 3 (2009) 266.

23 G. Janacek and L. Swift: Time Series Forecasting, Simulation, Applications (Ellis Horwood Limited, UK, 1993) p. 48.

24 B. Ninness and S. Gibson: The EM Algorithm for Multivariable Dynamic System Estimation (University of Newcastle, Australia, 2001) p. 1.

25 K. Lu and C. Hu: Electron. Opt. Control 1 (2010) 88.

26 Z. Zhou, C. Hu, and X. Han: Syst. Eng. Electron. 3 (2007) 416.

27 P. Li, J. Liu, J. Lai, and K. Huang: Int. J. Light Electron. Opt. 126 (2015) 2529. 